\title{
Genital Vulvar Lichen Sclerosus in Monozygotic Twin Women: A Case Report and Review of the Literature
}

\author{
Georgeta Doulaveri $^{\mathrm{a}} \quad$ Kalliopi Armira ${ }^{\mathrm{a}}$ Anargyros Kouris $^{\mathrm{a}}$ \\ Dimitrios Karypidis $^{\mathrm{b}}$ Irene Potouridou ${ }^{\mathrm{a}}$ \\ ${ }^{a}$ Department of Dermatology and Venereology, Hospital 'Andreas Sygros', \\ Athens, Greece; ${ }^{b}$ Wound Healing Research Unit, Cardiff University, Cardiff, UK
}

\section{Key Words}

Lichen sclerosus · Family · Twins

\begin{abstract}
Lichen sclerosus et atrophicus is a skin disease of multifactorial etiology which appears in patients with genetic or hormonal predisposition and autoimmune disease. Genetic predisposition is suggested by familial reports of the disease which involve twins, siblings, and mother-daughter/son series. This is a report of the occurrence of lichen sclerosus et atrophicus in monozygotic twin women, suggesting that inheritance is of relevance in the etiology of this disease.

(c) 2013 S. Karger AG, Basel
\end{abstract}

\section{Introduction}

Lichen sclerosus et atrophicus is a chronic disease of the skin and mucosa. It occurs at all ages, ranging from childhood to old age. Both sexes are affected, both before and after puberty, with female predominance at all ages. Postmenopausal women and prepubertal girls are most frequently affected $[1,2]$. The prevalence is about $1.7 \%$ in the general female population. The disease is characterized by white, polygonal papules, plaques, or vague, atrophic lesions. In atrophic lesions, the skin is thin, slightly wrinkled, soft, and white. Purpura, telangiectasias, and hemorrhagic blisters may occur. Additional symptoms are itching, which is frequently severe especially in the anogenital area, fissuring and erosion, which may result in dysuria, urethral and vaginal discharge, dyspareunia, and burning pain.

The pathogenesis of lichen sclerosus et atrophicus is poorly understood. Genetic factors are involved in the etiology of the disease based on the fact that there are reports of familial 
cases and association with HLA antigens. However, it has also been suggested that autoimmune mechanisms may be responsible because there is a well-established association between lichen sclerosus et atrophicus and autoimmune disease. In addition, environmental and hormonal factors are considered to be important. Although some cases of familial lichen sclerosus et atrophicus have been reported in the literature, it remains a rather understudied and uncommon type of lesion. However, it is of increasing interest due to its multidimensional clinical and pathophysiological characteristics. There have been reports of sibling pairs, twin pairs and mother-daughter/son associations. This is a rare report of 2 monozygotic twin sisters who had lichen sclerosus et atrophicus in the genital area, without any clear evidence of either a genetic or autoimmune basis, showing the investigative mystery that often accompanies this lesion.

\section{Case Report}

A.T. and O.T. are clinically identical twin women aged 37 years. At the age of 34 years, O.T. developed white atrophic patches on her vulvar skin, which extended onto the perineum. Itching and dyspareunia were the dominant symptoms (fig. 1, fig. 2). The clinical diagnosis was lichen sclerosus et atrophicus. A.T., at the age of 35 years, developed similar changes due to lichen sclerosus et atrophicus affecting her vulva and perineum. Neither sister had any involvement of nongenital skin. Their medical history was unremarkable. Physical examination of internal organs, gynecological examination, and Pap smears were normal for both sisters. Neither sister had any history of trauma in the genital region. Both sisters had had uneventful pregnancy and childbirth, and their children had no perinatal problems. Their family history indicated that their mother suffered from lichen sclerosus et atrophicus and their father from balanitis xerotica obliterans. The results of laboratory studies (blood count, vitamin D3, vitamin B12, iron, folic acid, ferritin, C-reactive protein, glucose, urea, creatinine, uric acid, cholesterol, triglycerides, high-density lipoprotein, lowdensity lipoprotein, serum glutamic oxaloacetic transaminase, serum glutamic-pyruvic transaminase, alkaline phosphatase, bilirubin, triiodothyronine, thyroxine, and thyroidstimulating hormone) and sex hormone level tests (follicle-stimulating hormone, luteinizing hormone, prolactin, and dehydroepiandrosterone sulfate) were normal. Antithyroid autoantibodies (anti-TPO and anti-Tg) were detected in serum from the twins and their parents. Moreover, only A.T. was positive for antinuclear antibodies. Antibodies to Borrelia were negative for both twins. The differential diagnosis involved genital lichen planus and lichen simplex chronicus. The diagnosis was supported by the skin biopsy specimen in the most mature area, which showed features of lichen sclerosus et atrophicus (fig. 3). The epidermis was thinned and the rete ridges were effaced. Compact hyperkeratosis and vacuolization of the focal basal cell layer were noted. A pale, widened papillary dermis with superficial perivascular and sparse diffuse lymphocytic infiltration of the upper reticular dermis were also observed.

The 2 women were treated with local steroids once a day for 2 months. Subsequently, ointment tacrolimus $0.1 \%$ was applied once a day for 2 months and then every other day for the next 2 months, with clinical improvement. Oral vitamin E $200 \mathrm{mg}$ was given once a day from the beginning of the treatment. At the end of the treatment and at the 6-month followup, clinical improvement was maintained and the lesions remained asymptomatic. 


\section{Discussion}

Lichen sclerosus et atrophicus is a chronic inflammatory disease of unknown etiology. A genetic susceptibility is suggested by the occurrence of familial cases. Sherman et al. [3] interviewed 1,052 individuals affected by lichen sclerosus and a family history was found in $12 \%$. These familial cases provide further evidence for the role of genetics in the etiology of lichen sclerosus et atrophicus.

There seems to be a clinical association between lichen sclerosus et atrophicus and autoimmune disease. Our case supports this theory because the twin women and their parents demonstrated changes in antithyroid autoantibodies, while 1 sister had positive antinuclear antibodies. In addition, their mother also suffered from lichen sclerosus et atrophicus and their father from balanitis xerotica obliterans.

In a study of 84 patients with lichen sclerosus et atrophicus, Marren et al. [4] revealed a high incidence of positive antithyroid, gastric parietal cell, intrinsic factor, antinuclear, and antismooth muscle autoantibodies. Powell et al. [5] found an increased frequency of HLADR4, DR11, DR12, DQ2, and DQ8. Gao et al. [6] observed an increased frequency of DRB1*12/DQB1*03 and a lowered frequency of DRB1*0301/04/DRB180201/02/03.

Aslanian et al. [7] found 8 proven cases of lichen sclerosus et atrophicus, and the most common HLA markers were B*15, B*57, CW*07, CW*18, DRB1*04, DRB1*07, and DRB4. They observed autoimmune phenomena in $40 \%$ of their patients, mainly autoimmune thyroid disease, vitiligo, pernicious anemia, rheumatoid arthritis, and alopecia areata. Purcell et al. [8] investigated 35 patients with lichen sclerosus et atrophicus and found an increased association with HLA-A29 and HLA-B44 which was statistically significant, while an increased incidence of HLA-B8 and HLA-DR3 was not statistically significant.

Holt and Darke [9], in 1983, revealed an association between HLA-AW31 and lichen sclerosus et atrophicus. The association of lichen sclerosus et atrophicus with HLA antigens and the strong clinical association with autoimmune phenomena suggest that it might be an immunologically mediated disease [4].

Our twin case suggests that environmental factors may also play an important role in the pathogenesis of lichen sclerosus et atrophicus. The development of the disease in identical twins is probably a reflection of exposure to the same environmental factor(s), while a genetic predisposition common to both twins exists $[7,10]$.

Hormonal factors may contribute to the pathogenesis of lichen sclerosus et atrophicus because the disease arises at times of relative estrogen deficiency. Friedrich and Kalra [11] demonstrated low levels of dihydrotestosterone, free testosterone and androstenedione as well as low activity of 5-a reductase. However, the serum sex hormone levels in our patients were normal.

Family studies contribute to a genetic factor in the etiology of lichen sclerosus et atrophicus. This may have implications for future treatment and for the prediction of the disease. Nevertheless, it is an exciting area and further research is needed, especially in cases where no current etiologic basis is evident.

\section{Disclosure Statement}

The authors declare no conflict of interest. 
Doulaveri et al.: Genital Vulvar Lichen Sclerosus in Monozygotic Twin Women: A Case Report and Review of the Literature

\section{References}

1 Murphy FR, Lipa M, Haberman HF: Familial vulvar dystrophy of lichen sclerosus type. Arch Dermatol 1982;118:329-331.

-2 Shirer JA Jr, Ray MC: Familial occurrence of lichen sclerosus et atrophicus. Case reports of a mother and daughter. Arch Dermatol 1987;123:485-488.

-3 Sherman V, McPherson T, Baldo M, et al: The high rate of familial lichen sclerosus suggests a genetic contribution: an observational cohort study. J Eur Acad Dermatol Venereol 2010;24:1031-1034.

4 Marren P, Yell J, Charnock FM, et al: The association between lichen sclerosus and antigens of the HLA system. Br J Dermatol 1995;132:197-203.

5 Powell J, Wojnarowska F, Winsey S, et al: Lichen sclerosus premenarche: autoimmunity and immunogenetics. Br J Dermatol 2000;142:481-484.

6 Gao XH, Barnardo MC, Winsey S, et al: The association between HLA DR, DQ antigens, and vulval lichen sclerosus in the UK: HLA DRB112 and its associated DRB112/DQB10301/04/09/010 haplotype confers susceptibility to vulval lichen sclerosus, and HLA DRB10301/04 and its associated DRB10301/04/DQB10201/02/03 haplotype protects from vulval lichen sclerosus. J Invest Dermatol 2005;125:895-899.

7 Aslanian F, Marques MT, Matos H, et al: HLA markers in familial lichen sclerosus. J Dtsch Dermatol Ges 2006;4:842-847.

-8 Purcell KG, Spencer LV, Simpson PM, et al: HLA antigens in lichen sclerosus et atrophicus. Arch Dermatol 1990;126:1043-1045.

-9 Holt PJ, Darke C: HLA antigens and Bf allotypes in lichen sclerosus et atrophicus. Tissue Antigens 1983;22:89-91.

10 Meyrick Thomas RH, Kennedy CT: The development of lichen sclerosus et atrophicus in monozygotic twin girls. Br J Dermatol 1986;114:377-379.

11 Friedrich EG Jr, Kalra PS: Serum levels of sex hormones in vulvar lichen sclerosus and the effect of topical testosterone. N Engl J Med 1984;310:488-491.

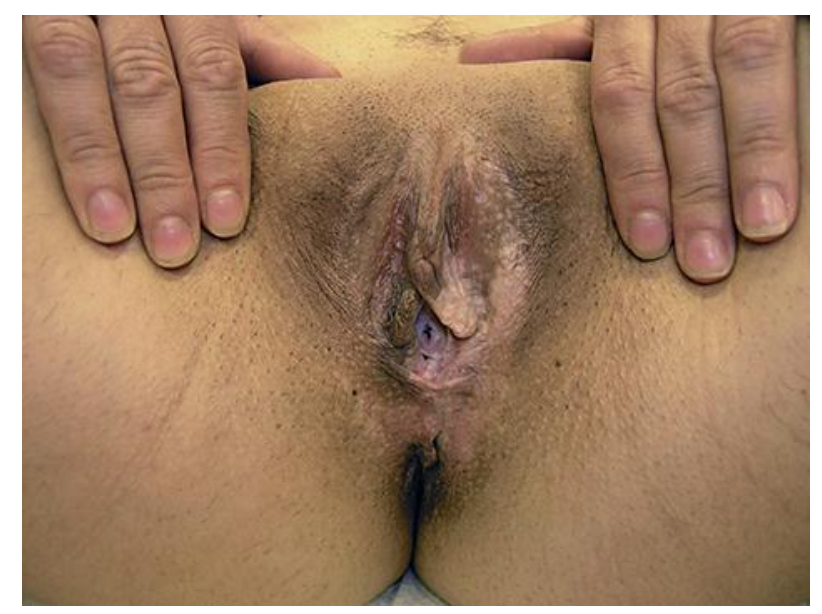

Fig. 1. White atrophic patches which extend onto the perineum in A.T. 
Doulaveri et al:: Genital Vulvar Lichen Sclerosus in Monozygotic Twin Women: A Case Report and Review of the Literature

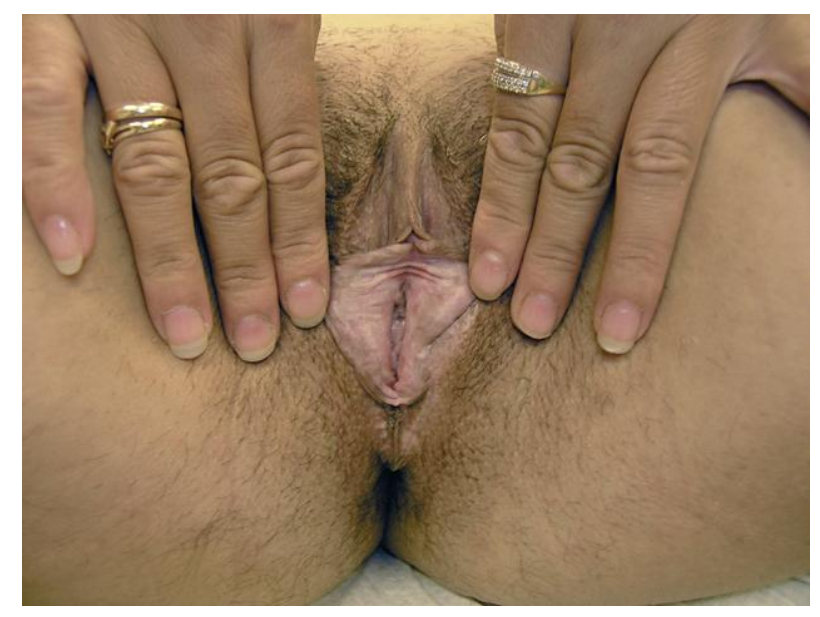

Fig. 2. White atrophic patches which extend onto the perineum in O.T.

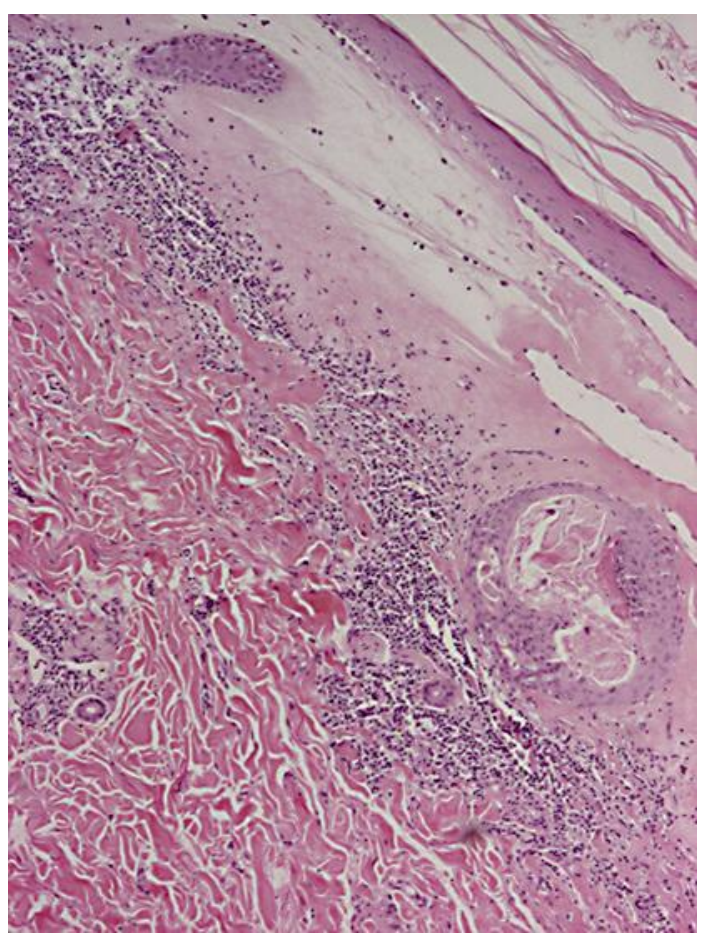

Fig. 3. Thinned epidermis, effaced rete ridges, vacuolization of the focal basal cell layer, and superficial perivascular and sparse diffuse lymphocytic infiltration of the upper reticular dermis. HE. $\times 4$. 